\title{
Nano-Electromechanical Systems: Displacement Detection and the Mechanical Single Electron Shuttle
}

\author{
R.H. Blick, F.W. Beil, E. Höhberger, A. Erbe, and C. Weiss \\ Center for NanoScience and Sektion Physik, Ludwig-Maximilians-Universität, \\ Geschwister-Scholl-Platz 1, 80539 München, Germany.
}

\begin{abstract}
For an introduction to nano-electromechanical systems we present measurements on nanomechanical resonators operating in the radio frequency range. We discuss in detail two different schemes of displacement detection for mechanical resonators, namely conventional reflection measurements of a probing signal and direct detection by capacitive coupling via a gate electrode. For capacitive detection we employ an on-chip preamplifier, which enables direct measurements of the resonator's displacement. We observe that the mechanical quality factor of the resonator depends on the detection technique applied, which is verified in model calculations and report on the detection of sub-harmonics. In the second part we extend our investigations to include transport of single electrons through an electron island on the tip of a nanomachined mechanical pendulum. The pendulum is operated by applying a modulating electromagnetic field in the range of $1-200 \mathrm{MHz}$, leading to mechanical oscillations between two laterally integrated source and drain contacts. Forming tunneling barriers the metallic tip shuttles single electrons from source to drain. The resulting tunneling current shows distinct features corresponding to the discrete mechanical eigenfrequencies of the pendulum. We report on measurements covering the temperature range from $300 \mathrm{~K}$ down to $4.2 \mathrm{~K}$. The transport properties of the device are compared in detail to model calculations based on a Master-equation approach.
\end{abstract}

\section{Introduction}

Nano-electromechanical systems (NEMS) are investigated because of their promising features regarding sensitive tools for sensor and communications technology. This systems may also be used as 'quantum-mechanical' resonators which allow to explore mechanics in the quantum mechanical range when operated at several $\mathrm{GHz}$ at ultra low temperatures. In the first part we want to address different detection schemes necessary to achieve sensitive displacement detection for NEMS. It is shown that the measured Q-value of this structures depends on the detection scheme used. This fact permits to increase the sensitivity of those systems by using the appropriate detection technique. In this way a handle on quantum squeezing experiments with these mesoscopic mechanical systems is attained [1]. This scheme of detection is also suited for probing quadrature squeezed states [2].

In the second part we will focus on a non-classical version of one of the traditional experiments in the electrodynamics class: Usually it is set up by two large capacitor plates and a metallized ball suspended in between the plates. 
Applying a constant voltage of some $100 \mathrm{~V}$ across the plates leads to the onset of periodic charge transfer by the ball bouncing back and forth, similar to a classical bell [3. The number of electrons transferred by the metallized ball in each revolution naturally depends on the volume of the metal, but can be estimated to be of the order of $10^{10}$. At an oscillation frequency of some $10 \mathrm{~Hz}$ up into the audible $\mathrm{kHz}$-range this gives a typical current of $1-10 \mu \mathrm{A}$. The question arising is whether such an experiment can be performed on the microscopic level in order to achieve a transfer not of a multitude but of only one electron per cycle of operation at frequencies of some $100 \mathrm{MHz}$. Indeed this can be achieved by simply scaling down the setup and applying a nanomechanical resonator. Such a device is of great importance for signal processing applications and for metrology, since it allows to transfer single electrons at radio frequencies and reduces cotunneling events at the same time.

\section{Fabrication of Nanoscale Device with Three-Dimensional Relief}

The beam-resonators are built up of freely suspended silicon beams, covered by an $50 \mathrm{~nm}$ resp. $100 \mathrm{~nm}$ thick conducting layer, close to which tapered electrodes are mounted sidewise. These electrodes may serve for capacitive excitation and detection purposes. During the fabrication process optic and electron lithographic steps are used first, to structure gates and an etch mask. Than the resonators are defined by succeeding steps of dry and wet etching. Electron micrographs of this structures are show in Fig. 6. Processing of the electron shuttle is more advanced, since two steps in electron beam lithography have to be performed. The mechanical pendulum itself is defined by etching in a combination of dry and wet etch steps. Alignment of the etch mask with respect to the metallic leads has to be accurate down to $10 \mathrm{~nm}$ in order to provide well defined tunneling contacts. In earlier work we ensured that the processing steps, which are also used in our present work, provide clean and tuneable tunneling contacts [4. A schematic drawing of the measurement setup is shown in Fig.6. The details of the techniques used to fabricate the nanoscale devices are reported in preceding work in more detail [567].

\section{Detecting Motion of Nanomechanical Resonators}

The resonators can be driven either by the capacitive forces of the electrodes, or by Lorentz forces, due to placement of the structures in an external magnetic field and inducing an alternating current along the length of the conducting metal on top of the beam. One way of detecting the amplitude of an oscillating motion is to take advantage of the amplitude dependent impedance $\hat{Z}_{\text {res }}$ of the resonator component, due to electro motive forces. This is easily measured with an usual networkanalyser $H P 8751 A$ together with a test-set in order to measure the S-parameters of the component. With the mentioned side-gates it 


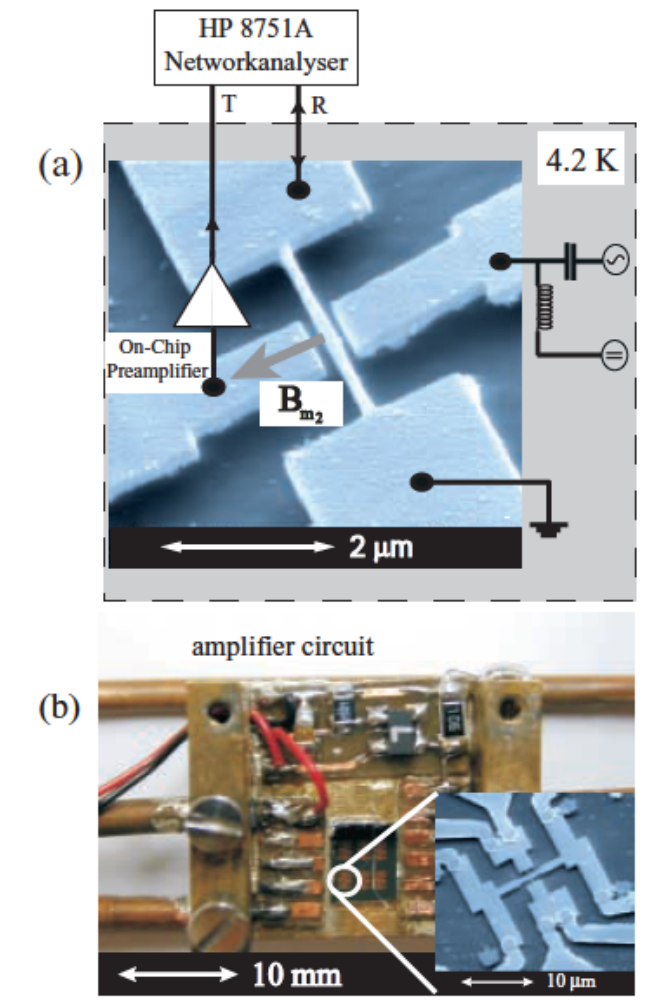

Fig. 1. (a) Scanning electron microscope micrograph of one of the nanomechanical resonators. The silicon beam is covered by a thin Au-sheet; the electrodes on the left and right allow application of an additional $a c$ voltage modulation. Also pictured is the experimental setup for sampling the mechanical properties of the suspended beam. For characterization we employ a network analyzer scanning the frequency $\omega$ over the range of interest (see text for details). (b) On-chip amplifier: One of the gates is coupled to the amplifier enabling capacitive detection of the beam's displacement. A magnified view of one of these is depicted in the inset.

is also possible to measure the mechanical displacement of the oscillating part capacitive. Due to the very small signal currents we used for this measurements a on-chip preamplifier, which was incorporated with the above mentioned highspeed field effect transistor, which served as an impedance-converter. An aerial view of the setup and the amplifier is depicted in Fig. 1. The magnetic field orientation was chosen to be parallel to the surface of the transistor. The total impedance of the beam resonator placed in a magnetic field $B$ is derived in [8]. This can be simplified assuming harmonic excitation at the eigenfrequency:

$$
\hat{Z}_{r e s}\left(\omega=\omega_{0}\right)=R+\frac{L^{2} B^{2}}{2 \mu m_{e f f}}
$$


where $m_{e f f}$ is the effective mass of the beam and the length of the beam $l$ is connected via $L=l \pi / 2$. The attenuation constant is referred to as $\mu$. The minimum detectable force using mechanical cantilevers is limited by the vibrational noise and is given by [8]

$$
F_{\text {min }} \approx \sqrt{\frac{\kappa}{\omega_{0}}} \approx \sqrt{\frac{w t^{2}}{l Q}},
$$

where $\kappa$ is the spring constant of the beam or cantilever, $w, t, l$, are the width, thickness, and length of the beam and $Q$ is the mechanical quality factor, being defined as $Q=f_{0} / \delta f_{0}$, where $\delta f_{0}$ denotes the width of the resonance peak. Regarding this context the aim is to achieve a considerable size reduction of the structures leading to higher eigenfrequencies.

Fig. 2 shows measured spectra of the reflected power for one of the two different magnetically driven beams with different dimensions (henceforth these resonators will be called $m_{1}$ and $m_{2}$, as assigned in Fig. 2). This resonators are for $4.2 \mathrm{~K}$ resonant at $95.93 \mathrm{MHz}$ for $m_{1} \operatorname{resp}$. $81.7 \mathrm{MHz}$ for $m_{2}$, where the varied magnetic field was oriented perpendicular to the beam for $m_{1}$ and parallel for $m_{2}$, so that two different modes, were generated. These resonances could be verified by capacitive detection. There are several factors which limit the quality of the resonator. The most important are losses due to the ohmic resistance of the conduction layers, the viscous inner damping of the material itself and the external resistance of the setup. The upper bound for the Q-value due to an finite resistance of the conduction layer can easily be estimated. Using this setup as an electrometer yields a charge sensitivity of $1.3 \times 10^{-3} \mathrm{e} / \sqrt{\mathrm{Hz}}$, which is three orders of magnitude better than previously measured [10].

The result when using capacitive detection to probe the resonances of $m_{2}$ is depicted in Fig. 3. In this measurement we observed an enhanced quality factor for $m_{2}$ of $Q \sim 4150$. This value is increased by a factor of 1.52 as compared to the value measured by the standard detection technique. This behaviour can be modeled by simply taking into account the different functional dependencies of the measuered signal on the excitation frequency [8]. The resonance has Lorenzian shape when measuring the induced voltage. Using capacitive detection the signal depends on the square of the Lorenzian, resulting in an enhanced qualityfactor and a shift of the peaks. The factor of 1.52 for the enhanced Q-value is reproduced by the calculations while the shift is observed but too large compared with theory.

To further increase the sensitivity of NEMS for sensor applications a simple approach is to probe harmonics of the base frequency at higher frequencies. Although, it is easy to calculate resonance frequencies by finite element methods (FEM), there has been no observation of several modes for a single structure so far. In Fig. 3 we present measurements on a third beam with sligthly modified dimensions revealing two Lorenzians. The measured spectrum in Fig. 4 shows two resonances at 26.9 MHz and 35.5 MHz. Using FEM 11 we can assign the resonance at $\omega_{0}=35.6 \mathrm{MHz}$ to the first eigenmode of the beam, which is also 


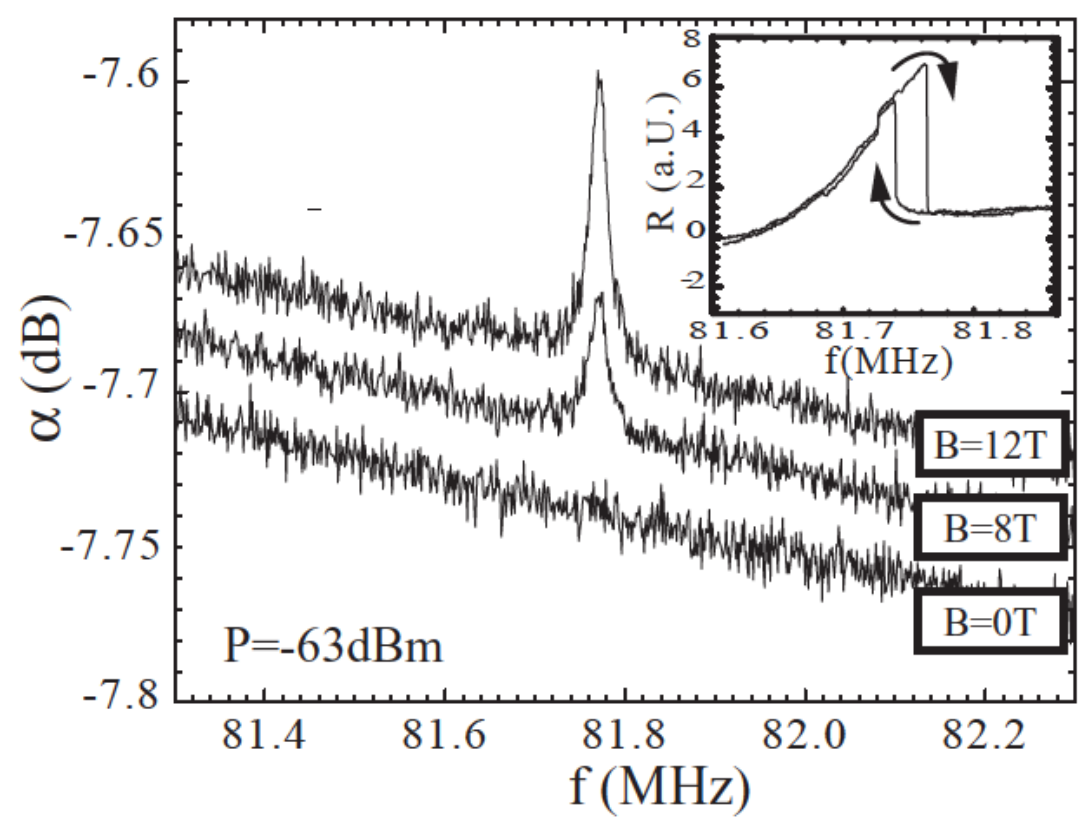

Fig. 2. Measured resonances for the two different beams. This curves where measured scanning the reflected power over the frequency-range of interest for different applied magnetic fields. This mechanical resonator is $4.8 \mu \mathrm{m}$ long, $170 \mathrm{~nm}$ width and $190 \mathrm{~nm}$ thick, covered by an $50 \mathrm{~nm}$ thick conducting Au layer. The irradiated power amounted $-63 d B$. The elongation of the structure is estimated to be about $10 \mathrm{~nm}$. The inset shows nonlinear effects observed when scanning the frequnecy range from low to high frequencies and reverse. In this case a hysteresis emerges.

depicted in the inset of Fig. 4. The second resonance at $27 \mathrm{MHz}$ can be explained by resonant excitation of a subharmonic at $\omega=3 / 4 \times \omega_{0}$. The inset of the Fig. 4 also shows the $Q$-values of the resonances for several temperatures.

\section{How to Shuttle Single Electrons Mechanically}

In this second part we want to focus on our results of ours on shrinking a mechanical electron shuttle to submicron dimensions by integration of an electron island into a nanomechanical resonator functioning as an electromechanical transistor (EMT) [12. The clear advantages are the increased speed of operation and the reduction of the transfer rate, allowing to count electrons one by one. This combination was proposed theoretically by Gorelik et al. [13] for metallic particles, which are connected to the reservoirs by elastically deformable organic molecu- 


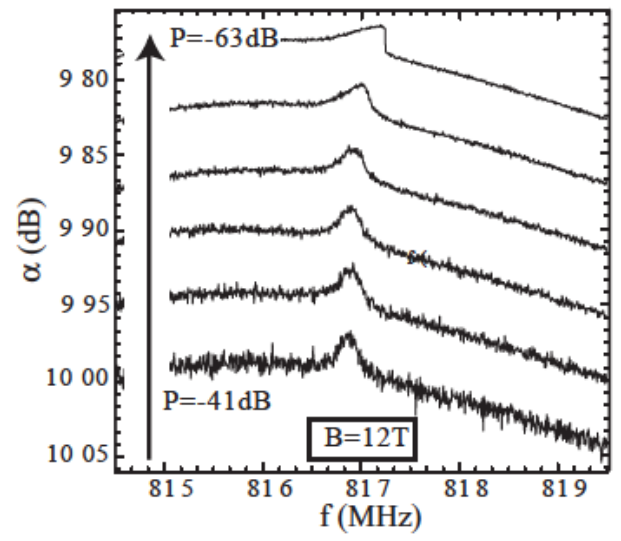

Fig. 3. Resonances in the spectrum of $m_{2}$ detected by capacitive coupling.

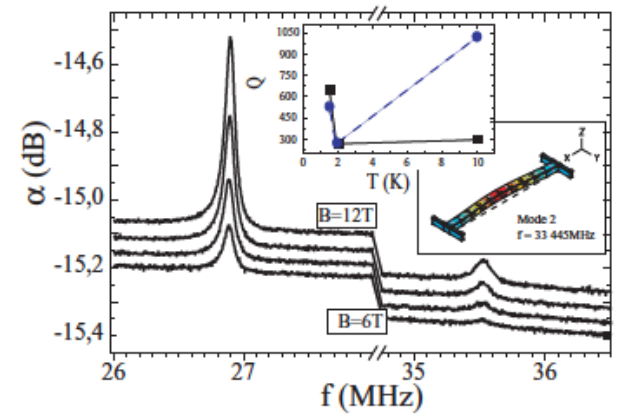

Fig. 4. Radio frequency spectrum of the reflected power for another resonator: Shown is the resonance of the base frequency $f_{0}=35.6 \mathrm{MHz}$ and a subharmonic at $3 / 4 \times f_{0}$. In the insets the calculated modeshape of the corresponding eigenmode is shown as well as the variation of the $Q$-value with temperature for the two peaks observed. The square dots refer to the resonance at $37 \mathrm{MHz}$ while the circular dots refer to the $35.6 \mathrm{MHz}$ peak.

lar links. The main difference to common SET devices is the fact that only one tunneling barrier is open at a certain time. This naturally leads to a suppression of cotunneling effects and thus increases the accuracy of current transport [14]. The reduced cotunneling effects may lead to accurate measurements of quantum fluctuations in these devices as well.

An important feature of the device is the possibility to effectively modulate the tunneling rate onto and off the electron island given by the large speed of operation $f \sim 100 \mathrm{MHz}$. This basically enables to mechanically filter and select electrons passing through the electromechanical circuit by simply adjusting the tunneling rate $\Gamma$. Additionally, the device is a tool to regularize the stochastic tunneling process through a well defined geometry. Another advantage of 
nanomechanical resonators, machined out of Silicon On Insulator (SOI) material, is their insensitivity to thermal and electrical shocks as has been shown in their application for electrometry [15[16]. This and the high speed of operation enable direct integration in filter applications [17. We have already demonstrated, that a nanomechanical tunneling contact, which operates at radio frequencies (rf), can be built out of SOI substrates [4. The tunneling process in turn is very sensitive to changes of the environmental conditions, thus its use in sensor applications.

A schematic drawing of the measurement setup is shown in Fig. 6. In order to guarantee clean tunneling contacts for the real device shown in the sample chamber was heated and pumped prior to the measurements. The first measurements were performed at room temperature. The sample was mounted in an evacuated sample holder with a small amount of helium gas added, to ensure thermal coupling. The $300 \mathrm{~K}$ trace shows a variety of resonances, where the source/drain current is increased due to the motion of the clapper. This behavior is well known from the measurements performed on the single tunneling barrier 4 .

In order to calculate the probability $p(m, t)$ to find $m$ additional electrons on the island at time $t$ a Master equation was used [14]. In our case the island oscillates mechanically $\left(x(t)=x_{\max } \sin (\omega t)\right)$ which leads to time dependent transition rates $\Gamma(m, t)$ at the leads. Collecting gain and loss terms the Master equation reads

$$
\begin{aligned}
\frac{d}{d t} p(m, t)= & -\left[\Gamma_{\mathrm{L}}^{(+)}(m, t)+\Gamma_{\mathrm{R}}^{(+)}(m, t)+\Gamma_{\mathrm{L}}^{(-)}(m, t)+\Gamma_{\mathrm{R}}^{(-)}(m, t)\right] p(m, t) \\
& +\left[\Gamma_{\mathrm{L}}^{(+)}(m-1, t)+\Gamma_{\mathrm{R}}^{(+)}(m-1, t)\right] p(m-1, t) \\
& +\left[\Gamma_{\mathrm{L}}^{(-)}(m+1, t)+\Gamma_{\mathrm{R}}^{(-)}(m+1, t)\right] p(m+1, t)
\end{aligned}
$$

with golden rule tunneling rates of the form [18]

$$
\Gamma=\frac{1}{e^{2} R} \frac{\Delta E}{1-\exp \left(-\frac{\Delta E}{k_{\mathrm{B}} T}\right)} .
$$

The time dependence of both $R$ and the capacitance $C$ in $\Delta E \propto \frac{e^{2}}{C}$ leads to

$$
\Gamma_{\mathrm{R}}^{(\mp)}(m, t)=g_{\mathrm{R}}(t) \Gamma_{\mathrm{R}, m}^{(\mp)}(t)
$$

Here

$$
g_{\mathrm{R}}(t)=\frac{R_{\mathrm{R}}\left(t_{\max }\right) C_{\mathrm{R}}\left(t_{\max }\right)}{R_{\mathrm{R}}(t) C_{\mathrm{R}}(t)}
$$

is a strongly varying function of time, which is dominated by the exponentially varying $R(t)$ ( $t_{\max }$ is the time where the island is at its closest point to the right electrode). For the rates $\Gamma_{\mathrm{R}, m}^{(\mp)}(t)$ whose time dependence is determined by that of the capacitance only, we take the standard result for tunneling rates when a 
voltage of $-V / 2$ is applied at the left electrode and a voltage of $V / 2$ is applied at the right electrode:

$$
\Gamma_{\mathrm{R}, m}^{(\mp)}(t)=\frac{1}{\tau} \frac{ \pm\left(m+\frac{C_{\mathrm{L}}(t) V}{e}\right)-\frac{1}{2}}{1-\exp \left[-\left( \pm\left(m+\frac{C_{\mathrm{L}}(t) V}{e}\right)-\frac{1}{2}\right) \frac{e^{2}}{C_{\Sigma}(t) k_{\mathrm{B}} T}\right]}
$$

where $\tau=R_{\mathrm{R}}\left(t_{\max }\right) C_{\mathrm{R}}\left(t_{\max }\right)$ and $C_{\Sigma}(t)=C_{\mathrm{R}}(t)+C_{\mathrm{L}}(t)$. For the left electrode the indices $R$ and $L$ have to be interchanged, $V$ has to be replaced by $-V$ and $x(t)$ by $-x(t)$.

In order to be able to solve the master equations analytically, we replace the function $g_{\mathrm{R}}(t)$ by a step function $\tilde{g}_{\mathrm{R}}(t)$ having the same height and area:

$\tilde{g}_{\mathrm{R}}(t) \equiv \begin{cases}0 & : t \leq t_{\max }-t_{0} \\ 1 & : \quad t_{\max }-t_{0}<t<t_{\max }+t_{0} \quad, \quad t_{0} \equiv \sqrt{\frac{\pi \lambda}{2 x_{\max }}} \frac{1}{\omega}\left(1+\frac{\lambda}{2 x_{0}}\right) . \\ 0 & : \quad t \geq t_{0}+t_{\max }\end{cases}$

The width $2 t_{0}$ corresponds to an effective contact time. As expected, the contact time decreases for increasing oscillation frequencies $\left(x_{\max } \gg \lambda, t_{\max } \gg t_{0}\right)$.

The solutions $p(m \mid n, t)$ of the Master equation can be used to calculate the probability $p_{\Delta}(m)$ that $m$ electrons are transferred:

$$
p_{\Delta}(n) \equiv \sum_{k} p_{\mathrm{i}}(k) p\left(k-n \mid k, t_{0}+t_{\max }\right)
$$

where $p_{\mathrm{i}}(k)$ is the initial probability to find $k$ additional electrons on the island $\left(\sum_{k} p_{\mathrm{i}}(k)=1\right)$. From $p_{\Delta}(n)$ we then calculated both $\langle N\rangle$ and $\Delta N\left(\left\langle N^{k}\right\rangle \equiv\right.$ $\left.\sum_{N} N^{k} p_{\Delta}(N)\right)$.

For very low temperatures $k_{\mathrm{B}} T \ll \frac{e^{2}}{C}$ Coulomb blockade fixes the number of additional electrons $n$ on the island between $-n_{\max }$ and $n_{\max }$ strictly. The resulting coulomb staircase is shown in Fig. 5.

In the middle of the first step the analytic expressions for the average number of electrons transferred $(\langle N\rangle)$ and the mean square fluctuations $\left((\Delta N)^{2}\right)$ are comparatively simple [14]:

$$
\begin{aligned}
\langle N\rangle & =2 \frac{1-a^{3}}{[1+a]\left[1+\frac{1}{2} a+a^{2}\right]} \\
(\Delta N)^{2} & =2 a(1-a) \frac{6+9 a+22 a^{2}+13 a^{3}+10 a^{4}}{\left[2 a^{2}+a+2\right]^{2}[a+1]^{2}}
\end{aligned}
$$

where $a \equiv \exp \left(-\frac{t_{0}}{\tau}\right)$.

In the present experiment [12] the clapper is set into motion by an $a c$-voltage applied to the two driving gates at frequency $f_{0}$. This leads to an alternating force acting on the grounded lower part of the pendulum. Additionally, the driving voltage also acts as a gate voltage. The gate capacitance is approximately 

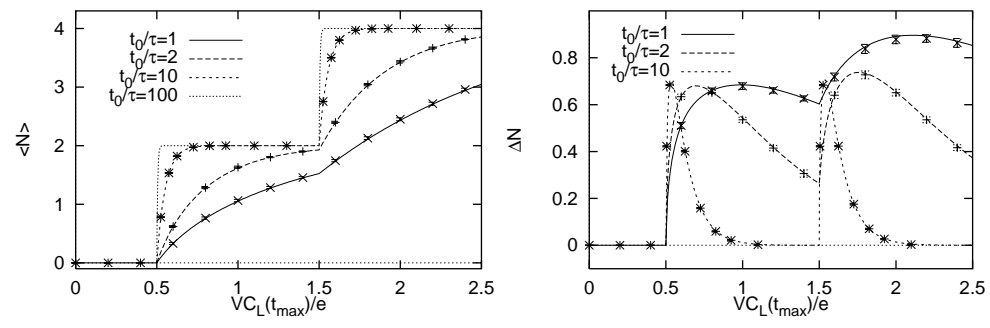

Fig. 5. The average number of electrons transferred per period (left) and the root mean fluctuations (right) for $T=0$. Coulomb blockade is clearly visible: up to a critical voltage $\left(\frac{V C_{\mathrm{L}}\left(t_{\max }\right)}{e}=\frac{1}{2}\right)$ no electrons are transferred. The Coulomb staircase becomes symmetric for $t_{0} \gg \tau$. The agreement between analytical results (lines) and computer simulations (crosses) is excellent [14].

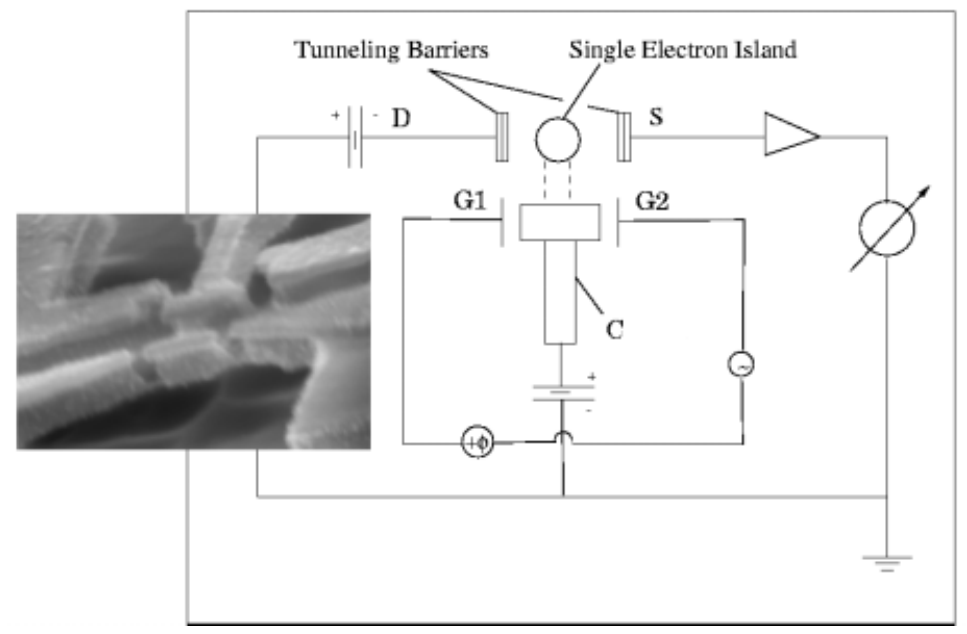

Fig. 6. Schematic drawing of the measurement setup. The single electron island is situated at the top of the clapper and supported by a silicon beam. The clapper is set into motion by applying an $a c$-voltage to the driving gates $G_{1}$ and $G_{2}$. The $a c$-voltage on the gates are phase shifted for optimal coupling of the $a c$-power to the motion of the beam. The $d c$-current from the source to the drain contact is measured with a current-voltage converter. The inset shoes an SEM-picture of the sample.

$C \approx 84 \mathrm{aF}$ which corresponds to gate charges of up to $\pm 527 e$ if voltages of up to $\pm 1 V$ are applied. We modified Eq. (3) in order to account for the influence of the gate voltage [12. If the frequency $f_{0}$ of the driving force meets the eigenfrequency of the clapper, resonant motion is excited. In our calculations this kind of motion shows a large number of electrons transferred at a high accuracy during each cycle. This creates the large peaks seen in the measurements. If the shuttle moves with frequencies different from $f_{0}$ (e.g. excited thermally), the resulting 
current has a much smaller signal to noise ratio. Therefore, the large value of the gate-voltage explains both the high number of electrons transferred at room temperature and the background depending on whether or not the clapper moves with the same frequency as the applied voltage.

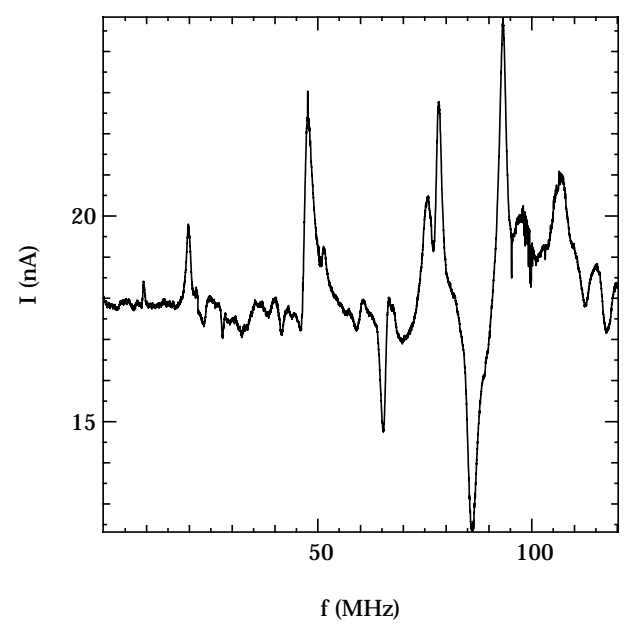

Fig. 7. Measurement of the tunnel current from source to drain at room temperature. The great number of peaks can be understood by assuming a complex mode spectrum. This fact is supported by numerical calculations using a finite element program [11.

Numerical solution of the modified Eq. (3) results in the height of the peaks being determined by the applied gate-voltage $V_{\mathrm{G}}$. The number of electrons transferred does hardly depend on source/drain bias, since the voltages applied on the driving gates are a factor $10^{3}$ larger than the source/drain bias. In the experiment the peaks are superimposed on a background, which depends linearly on the source/drain bias. This background is due to the thermal motion of the clapper, since it disappears at lower temperatures. The displacement noise of a cantilever can be calculated in analogy to an electrical circuit [19. The response of the clapper to an external force was calculated by a finite element program [11]. We conclude from this calculation, that contributions to the current can be expected up to frequencies of some GHz. This explains the amplitude of the thermal background.

The peak height does not depend on the $d c$-source/drain bias as well. Both facts can be explained by the large gate voltage.

The number of transferred electrons in this peak is $\approx 1000$ which agrees with our theoretical estimate based on Eq. (3). These results have shown, that the electron transfer works well at room-temperature. Since electron tunneling should be very sensitive to environmental influences this opens an interesting possibility for sensor applications. 
Measurements at lower temperatures show a complete suppression of the ohmic background and thus indicate its thermal nature. At temperatures of about $12 \mathrm{~K}$ a pronounced peak at $120 \mathrm{MHz}$ is found. This peak is strongly attenuated towards lower temperatures of $4.2 \mathrm{~K}$ due to the increased stiffness of the clapper. The rest of the complex spectrum is completely suppressed at $4.2 \mathrm{~K}$ also because of the increased stiffness of the structure.

At helium temperature the motion of the clapper is strongly reduced, resulting in very high tunnel barriers at the turning points. The maximum current amplitude of the peak is $(2.3 \pm 0.02) \mathrm{pA}$, which corresponds to a transfer of 0.11 electrons on average per cycle of motion of the clapper. Peaks in the classical experiment [4] show Lorentzian line shape. In order to obtain a formula for the peak fit for the present experiment we use the expression 10 in the limit of low contact times. Combined with the well known equations for the damped harmonic oscillator

$$
x^{\prime \prime}(t)+2 \pi k x^{\prime}(t)+4 \pi^{2} f_{0}^{2} x(t)=F \sin (2 \pi f t)
$$

we obtain 12

$$
N=\frac{A}{f} \sqrt{\frac{x_{\max }\left(f_{\mathrm{r}}\right)}{x_{\max }(f)}} \exp \left[-B\left\{1-x_{\max }(f) / x_{\max }\left(f_{\mathrm{r}}\right)\right\}\right]
$$

where $x_{\max }(f) \propto 1 / \sqrt{\left(f^{2}-f_{0}^{2}\right)^{2}+k^{2} f^{2}}$ is the amplitude of the oscillation in resonance and $f_{r}=\frac{f_{0}}{2} \sqrt{4-2 k}$ is the shifted frequency of the damped oscillator, respectively. From the fit parameters we obtain a quality factor $Q=\frac{f_{0}}{k}$ of order 10. Small $Q$ s are essential for operation as a switch where the oscillating force in Eq. (12) is replaced by a step function. For small quality factors the oscillatory solution of the differential equation vanishes on a short timescale.

The shape of the resonance differs strongly from the measurements on a single tunnelbarrier shown in Fig.8 b). In these measurements the peak shape could be modelled by a Lorentzian line shape [4.

\section{Summary}

We have presented measurements on several nanomachined resonators operating in the radio frequency regime. An on-chip preamplifier enables us to detect the displacement of the nanowires directly by capacitive coupling. This is compared to the conventional method which monitors the reflection of incident power. We find changes in the $Q$-factors depending on the detection scheme applied. This dependence can be modelled when taking into account the difference in dependence of the signals on the amplitude of oscillation. We also found evidence for the generation of sub-harmonic resonances of the nanomachined beam studied. This will allow to further increase the force sensitivity, by pumping the nanomechanical system on the fundamental mode while probing on one of the harmonic modes. 
a)

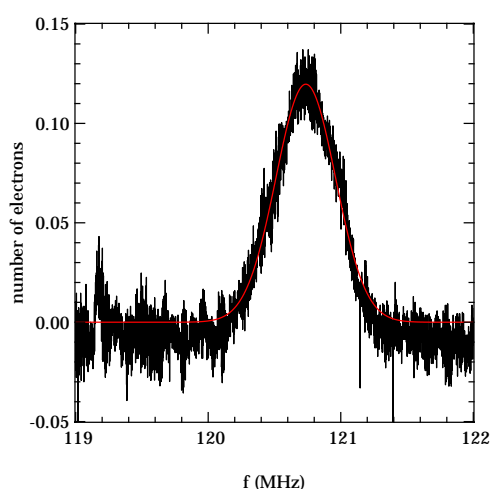

b)

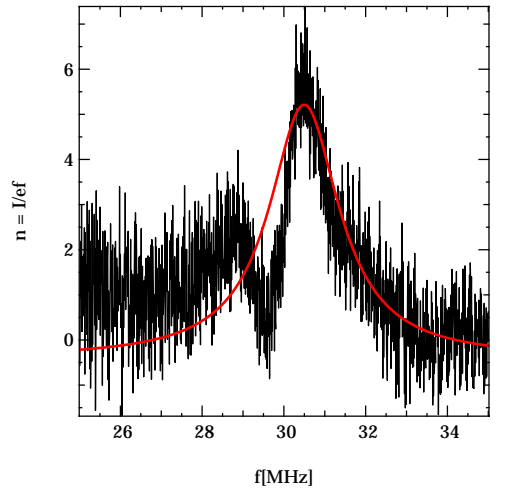

Fig. 8. a) At low temperatures only one peak remains, which can be fitted by the model given in Eq. (13). b) For comparison we show a peak measured on a single mechanically moving tunneling contact 4 .

In further experiments on mechanical resonators we have shown single electron tunneling by using a combination of nanomechanics and single electron devices. We have demonstrated a new way to transfer electrons one by one at radio frequencies. At $4.2 \mathrm{~K}$ we measured an average of $0.11 \pm 0.001$ electrons which shows that the resolution of current transport through the shuttle should also resolve Coulomb blockade effects. We estimate the temperature, at which Coulomb blockade should be observable to be $600 \mathrm{mK}$. Scaling down the island size will increase this temperature.

\section{Acknowledgements}

We like to thank J.P. Kotthaus and W. Zwerger for support and M.L. Roukes and H. Pothier for detailed discussions. We acknowledge financial support by the Deutsche Forschungsgemeinschaft under contracts DFG-Bl-487/1-1 and DFGBl-487/3-1.

\section{References}

1. X. Hu and F. Nori, Phys. Rev. B 53, 2419 (1996).

2. D. Rugar and P. Grütter, Phys. Rev. Lett. 67, 699 (1991).

3. P. Benjamin, The Intellectual Rise in Electricity (Appleton, New York, 1895), p. 507.

4. A. Erbe, R.H. Blick, A. Tilke, A. Kriele, and J.P. Kotthaus, Appl. Phys. Lett. 73, 3751 (1998).

5. H. Krömmer, A. Erbe, A. Tilke, S.M. Manus, and R.H. Blick, Europhys. Lett. 50, 101 (2000). 
6. L. Pescini, A. Tilke, R.H. Blick, H. Lorenz, J.P. Kotthaus, W. Eberhardt, and D. Kern, Nanotechnology 10, 418 (1999).

7. A. Kraus, A. Erbe, R.H. Blick, Nanotechnology 11, 165 (2000); A. Kraus, Diploma thesis, Ludwig-Maximilians-Universität, Munich, Germany 2000.

8. F.W. Beil, A. Kraus, A. Erbe, E. Höhberger, and R.H. Blick, "Approching the limits of displacement in nanomechanical resonators", preprint (2000).

9. T.D. Stowe, K.Yasumura, T.W. Kenny, D.Botkin, K. Wago, D. Rugar, Appl. Phys. Lett. 71, 288 (1997).

10. A.N. Cleland and M.L. Roukes, Nature 392, 160 (1998).

11. solvia, v. 95.2 (a finite element system).

12. A. Erbe, C. Weiss, W. Zwerger, and R.H. Blick, "A nanomechanical resonator shuttling single electrons at radio frequencies", preprint (2000).

13. L.Y. Gorelik, A. Isacsson, M.V. Voinova, R.I. Shekter, and M. Jonson, Phys. Rev. Lett. 80, 4526 (1998)

14. C. Weiss and W. Zwerger, Europhys. Lett, 47, 97 (1999).

15. A.N. Cleland and M.L. Roukes, Appl. Phys. Lett. 69, 2653 (1996).

16. H. Kroemmer, A. Erbe, A. Tilke, S.M. Manus, and R.H. Blick, Europhys. Lett. 50, 101 (2000).

17. N.M. Nguyen and R. G. Meyer, IEEE J. of Solid-State Circuits, SC-25, no. 4, 1028 (1990).

18. Single Charge Tunneling edited by H. Grabert and M. H. Devoret, NATO ASI Ser. B. 294, Plenum, New York (1992).

19. G. G. Yaralioglu and A. Atalar, Rev. Sci. Instr. 70, 2379 (1999). 